PAPers in Physics, VOL. 13, ART. 130003 (2021)

Received: 17 February 2021, Accepted: 17 April 2021

Edited by: O. Fojón

Reviewed by: D. K. Nguyen, Thu Dau Mot University, Vietnam

P. F. Weck, University of Nevada, Las Vegas, USA

Licence: Creative Commons Attribution 4.0

DOI: https://doi.org/10.4279/PIP.130003

www.papersinphysics.org

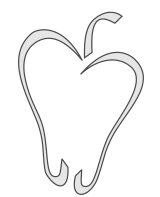

ISSN 1852-4249

\title{
An $a b$ initio study of small gas molecule adsorption on the edge of N-doped sawtooth penta-graphene nanoribbons
}

\author{
Nguyen Thanh Tien ${ }^{1 *}$, Le Vo Phuong Thuan ${ }^{1}$, Tran Yen $\mathrm{Mi}^{1}$
}

\begin{abstract}
Adsorption of the toxic gas molecules carbon monoxide $(\mathrm{CO})$, carbon dioxide $\left(\mathrm{CO}_{2}\right)$ and ammonia $\left(\mathrm{NH}_{3}\right)$ on the edge of $\mathrm{N}$-doped sawtooth penta-graphene nanoribbons (N:SSPGNRs) was studied using first-principles methods. Basing our study on density functional theory (DFT), we investigated adsorption configurations, adsorption energy, charge transfer, and the electronic properties of $\mathrm{CO}-, \mathrm{CO}_{2}$ - and $\mathrm{NH}_{3}$ - adsorbed onto $\mathrm{N}$ :SSPGNRs. We found that $\mathrm{CO}$ and $\mathrm{CO} 2$ are chemisorbed on the edge of N:SSPGNR, while NH3 is physisorbed. Current-voltage (I-V) characteristics were also investigated using the non-equilibrium Green's function (NEGF) approach. Gas molecules can modify the current of a device based on N:SSPGNRs. The results indicate the potential of using $\mathrm{N}$ :SSPGNRs for detection of these toxic gas molecules.
\end{abstract}

\section{Introduction}

Detecting gas molecules using semiconductor gas sensors is important for agriculture, chemical controls, environmental monitoring, and medical applications $[1,2]$. Low-dimensional systems, especially material systems based on graphene, have for many years demonstrated outstanding developments in sensors and transistor applications [3-8]. However, since the band gap of graphene is almost equal to 0 , it has not been fully exploited in the semiconductor industry [9]. The immense success of graphene [10-12] was followed by in-depth studies and encouraging efforts to find other twodimensional (2D) nanostructures, such as silicene [13], phosphorene [14] single-layer graphitic zinc oxide [15], h-boron nitride [16], and transition-metal dichalcogenides [17]. Penta-graphene (PG), a novel wide band gap carbon allotrope, and PG-like mate-

\footnotetext{
*nttien@ctu.edu.vn

1 College of Natural Sciences, Can Tho University, 3-2 Road, Can Tho City 94115, Vietnam
}

rials were discovered in early 2015 [18-20]. It was found that PG is ultra-strong, and can sustain a temperature of $1000 \mathrm{~K}$ with grain boundaries. It displays a quasi-direct intrinsic band gap of $3.2 \mathrm{eV}$ $[18,21]$. Additionally, using the $\mathrm{G}_{0} \mathrm{~W}_{0}$ approximation, the PG band gap was calculated as $4.2 \mathrm{eV}$ [22]. In contrast to hydrogenated graphene, hydrogenation of PG leads to a notable increase ( $76 \%$ rise) in thermal conductivity instead of the $63 \%$ reduction expected due to heat dispersal in device operation [23]. Furthermore, PG is noteworthy due to its unique mechanical properties and anisotropic mechanical behavior [24]. Unlike graphene, PG has a buckle structure, which allows it to adsorb gas molecules in rich configurations. Thus, PG is considered an excellent base for the development of gas sensors which can detect harmful gases such as $\mathrm{CO}$, $\mathrm{CO}_{2}$ and $\mathrm{NH}_{3}$ [25-27].

Cutting 2D-PG sheets in various crystallography directions obtains more different penta-graphene nanoribbons (PGNRs). Z. Q. Fan et al. found that the sawtooth-sawtooth PGNR (SSPGNR) is a more stable structure than the other three PGNR struc- 
a)

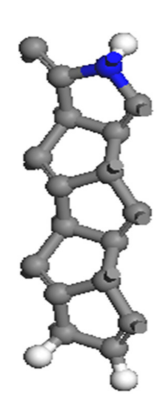

b)

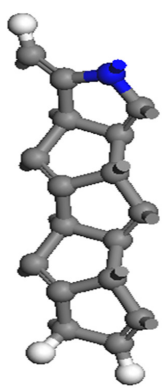

c)

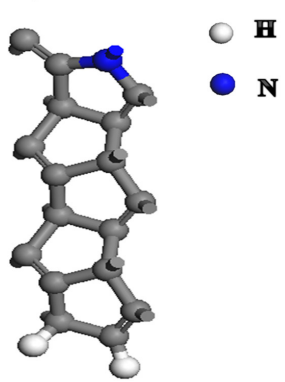

Figure 1: Schematic of possible N:SSPGNR adsorbent sites for gas molecules, consisting of a) C-H$\mathrm{N}: S S P G N R$, b) H-N-N:SSPGNR, c) C-N-N:SSPGNR.

tures [28]. PGNR receives major attention in semiconductor material science since its energy band gap can be controlled effectively in many ways, such as by applying an electric field and bending [29], doping [30,31], and edge termination [32,33]. In our previous studies [31] we discovered that the current intensity of N:SSPGNR increases to about $10^{8}$ times that of pure SSPGNR. This is convenient for determining current strength in electronic devices, including sensors. Furthermore, we studied the adsorption feature of the gas molecules $\left(\mathrm{CO}, \mathrm{CO}_{2}\right.$ and $\mathrm{NH}_{3}$ ) on the SSPGNR surface [34]. The results confirm that SSPGNR is sensitive to $\mathrm{CO}$ and $\mathrm{NH}_{3}$ molecules, but less sensitive to the $\mathrm{CO}_{2}$ molecule $[26,27]$. However, it is important to study the absorption configurations at the edges of nanoribbons $[8,35]$. SSPGNR can provide prior adsorption sites at its edges, which can serve as ideal gas sensor materials. Nitrogen doping at the carbon edge of PGNRs allows prior adsorption at this edge without hydrogen passivation. The carbon at the edge without hydogen passivation presents the dangling effect that favorably adsorbs $\mathrm{CO}, \mathrm{CO}_{2}$ and $\mathrm{NH}_{3}$. In this study, using DFT and NEGF methods we investigate theoretically the electronic and transport properties of N:SSPGNR when gas molecules (CO, $\mathrm{CO}_{2}$ and $\mathrm{NH}_{3}$ ) are adsorbed on their edge.

The paper is organized as follows: the subject and research objectives are presented in the introduction section; in section II the computational methods are discussed; section III contains the results and discussion of the electronic and transport properties of N:SSPGNR with the adsorbed

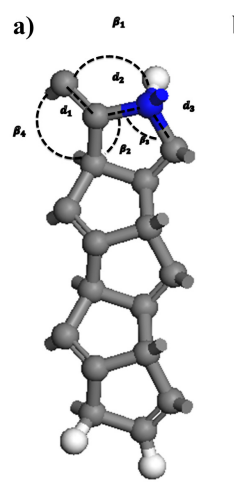

C $\bigcirc$ O b)

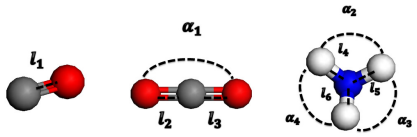

c)

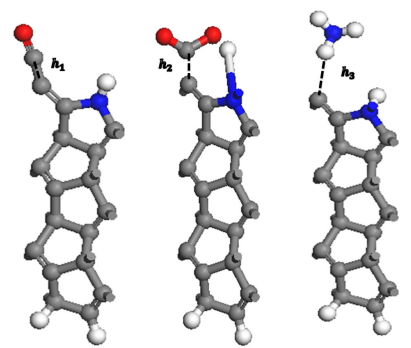

Figure 2: Adsorption configurations of C-HN:SSPGNR consist of: a) C-H-N:SSPGNR, b) isolated gas molecules and c) configurations of C-H-N:SSPGNR after adsorption gas molecules.

gas molecules on the edge; in section IV the conclusions are presented.

\section{Computational Methods}

The electronic and transport properties of $\mathrm{N}$ :SSPGNRs which adsorb gas molecules were explored by first-principles calculations based on DFT and NEGF, using the Atomistix ToolKit (ATK) software package (version 2017.1) [36,37]. The width of the studied structure was six sawtooth chains. A $10 \AA$ vacuum space was introduced along non periodic (i.e., $\mathrm{x}$ and $\mathrm{y}$ ) directions to ensure the isolation of N:SSPGNRs from their periodic replicas.

The samples were optimized using DFT calculations within the generalized gradient approximation (GGA) of Perdew Burke Ernzerhof (PBE) [40], with the following similar conditions: 1 x 1 x 9 kpoint sampling, a cut-off energy of $790 \mathrm{eV}$ and electron temperature of $300 \mathrm{~K}$. Considering the electric polarization effect, a double-zeta-polarized basis set was used to expand the electron wave function. The self-consistent field tolerance was set as $10^{-6} \mathrm{Ha}$. Furthermore, the ground state configuration was obtained at the convergence precision of energy for the maximum energy change, the maximum force, the maximum stress and the maximum displacement of $2.10^{-5} \mathrm{eV} /$ atom, $0.05 \mathrm{Ha} / \AA, 0.05 \mathrm{eV}^{-3}$ and $0.005 \AA$, respectively. 
Papers in Physics, vol. 13, ART. 130003 (2021) / N. T. Tien et al.

Table 1: Adsorption orientations and corresponding numbers for the adsorption structures of $\mathrm{CO}, \mathrm{CO}_{2}$ and $\mathrm{NH}_{3}$ on the edge of N:SSPGNR

\begin{tabular}{|c|c|c|c|}
\hline Gas & $\begin{array}{l}\text { Configurations } \\
\text { of N:SSPGNR }\end{array}$ & Adsorption orientations & Notation \\
\hline \multirow[t]{8}{*}{$\mathrm{CO}$} & \multirow[t]{2}{*}{ C-H-N:SSPGNR } & Vertical to $\mathrm{C}$ and $\mathrm{O}$ is downward & COCH1 \\
\hline & & Vertical to $\mathrm{C}$ and $\mathrm{O}$ is upward & $\mathrm{COCH} 2$ \\
\hline & \multirow{2}{*}{ H-N-N:SSPGNR } & Vertical to $\mathrm{N}$ and $\mathrm{O}$ is downward & COHN1 \\
\hline & & Vertical to Nintro and $O$ is upward & COHN2 \\
\hline & \multirow[t]{4}{*}{ C-N-N:SSPGNR } & Vertical to $\mathrm{C}$ and $\mathrm{O}$ is downward & COCN1 \\
\hline & & Vertical to $\mathrm{C}$ and $\mathrm{O}$ is upward & $\mathrm{COCN} 2$ \\
\hline & & Vertical to $\mathrm{N}$ and $\mathrm{O}$ is downward & COCN3 \\
\hline & & Vertical to $\mathrm{N}$ and $\mathrm{O}$ is upward & COCN4 \\
\hline \multirow[t]{12}{*}{$\mathrm{CO}_{2}$} & \multirow[t]{3}{*}{ C-H-N:SSPGNR } & $\begin{array}{l}\text { Vertical to } \mathrm{C} \\
\end{array}$ & $\mathrm{CO} 2 \mathrm{CH} 1$ \\
\hline & & Horizontal to $\mathrm{C}$ and $\mathrm{CO}_{2}$ perpendicular to $\mathrm{PG}$ & $\mathrm{CO} 2 \mathrm{CH} 2$ \\
\hline & & Horizontal to $\mathrm{C}$ and $\mathrm{CO}_{2}$ parallel to $\mathrm{PG}$ & $\mathrm{CO} 2 \mathrm{CH} 3$ \\
\hline & \multirow[t]{3}{*}{ H-N-N:SSPGNR } & Vertical to $\mathrm{N}$ & $\mathrm{CO} 2 \mathrm{HN} 1$ \\
\hline & & Horizontal to $\mathrm{N}$ and $\mathrm{CO}_{2}$ perpendicular to $\mathrm{PG}$ & $\mathrm{CO} 2 \mathrm{HN} 2$ \\
\hline & & Horizontal to $\mathrm{N}$ and $\mathrm{CO}_{2}$ parallel to $\mathrm{PG}$ & $\mathrm{CO} 2 \mathrm{HN} 3$ \\
\hline & \multirow[t]{6}{*}{ C-N-N:SSPGNR } & Vertical to $\mathrm{C}$ & $\mathrm{CO} 2 \mathrm{CN} 1$ \\
\hline & & Horizontal to $\mathrm{C}$ and $\mathrm{CO}_{2}$ perpendicular to $\mathrm{PG}$ & $\mathrm{CO} 2 \mathrm{CN} 2$ \\
\hline & & Horizontal to $\mathrm{C}$ and $\mathrm{CO}_{2}$ parallel to $\mathrm{PG}$ & $\mathrm{CO} 2 \mathrm{CN} 3$ \\
\hline & & Vertical to $\mathrm{N}$ & $\mathrm{CO} 2 \mathrm{CN} 4$ \\
\hline & & Horizontal to $\mathrm{N}$ and $\mathrm{CO}_{2}$ perpendicular to $\mathrm{PG}$ & $\mathrm{CO} 2 \mathrm{CN} 5$ \\
\hline & & Horizontal to $\mathrm{N}$ and $\mathrm{CO}_{2}$ parallel to $\mathrm{PG}$ & $\mathrm{CO} 2 \mathrm{CN} 6$ \\
\hline \multirow[t]{8}{*}{$\mathrm{NH}_{3}$} & \multirow[t]{2}{*}{ C-H-N:SSPGNR } & Gas molecule is downward & NH3CH1 \\
\hline & & Gas molecule is upward & $\mathrm{NH} 3 \mathrm{CH} 2$ \\
\hline & \multirow[t]{2}{*}{ H-N-N:SSPGNR } & Gas molecule is downward & NH3HN1 \\
\hline & & Gas molecule is upward & NH3HN2 \\
\hline & \multirow[t]{4}{*}{ C-N-N:SSPGNR } & Gas molecule is downward & NH3CN1 \\
\hline & & Gas molecule is upward & $\mathrm{NH} 3 \mathrm{CN} 2$ \\
\hline & & Gas molecule is downward & NH3CN3 \\
\hline & & Gas molecule is upward & NH3CN4 \\
\hline
\end{tabular}

\section{Results and Discussion}

\section{i Structure Stability}

To gauge the capacity of N:SSPGNRs to detect gas molecules $\left(\mathrm{CO}, \mathrm{CO}_{2}\right.$ and $\left.\mathrm{NH}_{3}\right)$, the adsorption of gas molecules was investigated on their edges, as the edge is the most reactive site on the ribbon due to the presence of dangling bonds.

Figure 1 depicts three possible adsorbent sites on the edge of an N:SSPGNR, including: a) removing a passive $\mathrm{H}$ atom at the top of the $\mathrm{C}$ atom (C$\mathrm{H}-\mathrm{N}$ :SSPGNR), b) removing a passive $\mathrm{H}$ atom at the top of the $\mathrm{N}$ atom (H-N-N:SSPGNR), and c) removing both passive $\mathrm{H}$ atoms on the edge of the ribbon (C-N-N:SSPGNR). To explore the preferred configuration, we had to identify the model which the guest molecules would approach uninhibitedly. We in turn determined the most appropriate configuration for each gas molecule: $\mathrm{CO}, \mathrm{CO}_{2}$ and $\mathrm{NH}_{3}$.
We first considered possible adsorption configurations of the $\mathrm{CO}$ molecule on the edge of an $\mathrm{N}$ :SSPGNR; CO can be adsorbed vertically on the edge with the $\mathrm{O}$ atom either upward or downward. Therefore, there are 8 possible adsorption configurations with the $\mathrm{CO}$ molecule. Similarly, there are 12 possible adsorption configurations for the $\mathrm{CO}_{2}$ molecule and 8 for the $\mathrm{NH}_{3}$ molecule. These configurations are listed in Table 1.

To determine the preferred configuration, we calculated the adsorption energy $\left(\mathrm{E}_{a d}\right)$ of all the configurations considered, as follows [38,39]:

$$
E_{a d}=E_{\text {total }}-E_{\text {ribbon }}-E_{\text {gas }},
$$

where $\mathrm{E}_{\text {total }}, \mathrm{E}_{\text {ribbon }}$ and $\mathrm{E}_{\text {gas }}$ are the total energies of a considered configuration after gas molecule adsorption, removing a passive $\mathrm{H}$ atom nanoribbon and isolated gas molecules, respectively. As per the definition adopted here, negative adsorp- 
tion energy shows that the process is exothermic in nature while the magnitude signifies thermodynamic stability. Computed results indicated that $\mathrm{COCH} 2, \mathrm{CO} 2 \mathrm{CH} 2$ and $\mathrm{NH} 3 \mathrm{CH} 2$ (Fig. 2) were the preferred configurations of $\mathrm{CO}, \mathrm{CO}_{2}$ and $\mathrm{NH}_{3}$ on N:SSPGNRs, respectively. The adsorption energy of these samples decreased gradually from -0.26 to $-2.96 \mathrm{eV}$ in the following order: $\mathrm{E}_{a d}\left(\mathrm{NH}_{3}\right)>$ $\mathrm{E}_{a d}\left(\mathrm{CO}_{2}\right)>\mathrm{E}_{a d}(\mathrm{CO})$. It is obvious that $\mathrm{CO}$ adsorption is the most stable.

In all three adsorption cases, the N:SSPGNR edge atom closest to gas molecules is a $\mathrm{C}$ atom rather than an $\mathrm{N}$ atom. This suggests that the most effective N:SSPGNR configuration for adsorption of gas molecules is the C-H-N:SSPGNR configuration. The adsorption distances of $\mathrm{CO}, \mathrm{CO}_{2}$, and $\mathrm{NH}_{3}$ are $1.291 \AA, 1.487 \AA$, and $3.317 \AA$, respectively, as shown in Table 2. Therefore, $\mathrm{CO}-$ and $\mathrm{CO}_{2-}$ adsorbed N:SSPGNRs are more capable of chemical adsorption than physical adsorption, while an $\mathrm{NH}_{3}$ adsorbed N:SSPGNR is more likely to adsorb physically [41].

\section{ii Parameters of structures}

Table 3 presents the parameters of three adsorption structures after relaxation. It can be clearly seen that the bond lengths of the gas molecules vary slightly. Namely, after the adsorption, the bond lengths of two adsorbed gas molecules $\left(\mathrm{CO}, \mathrm{CO}_{2}\right)$ are longer than those of isolated gas molecules. The opposite is true of $\mathrm{NH}_{3}$ gas molecules. In addition, the bond angle of the $\mathrm{CO}_{2}$ molecule is reduced from $180^{\circ}$ to $122^{\circ}$. The bond angles of the $\mathrm{NH}_{3}$ gas molecules change negligibly. In particular, the bond length of the CO adsorption sample changes the most. This is also one reason the adsorption energy in this sample is the largest. On the other hand, the bond lengths $\left(d_{1}, d_{2}, d_{3}\right)$ and

Table 2: Adsorption energy $\left(\mathrm{E}_{a d}\right)$, adsorption distance $(h)$, band gap $\left(\mathrm{E}_{g}\right)$ and charge transfer $(\mathrm{Q})$ from gas molecules to C-H-N:SSPGNR.

\begin{tabular}{lcccc}
\hline \hline Samples & $\begin{array}{c}\mathrm{E}_{a d} \\
(\mathrm{eV})\end{array}$ & $\begin{array}{c}\mathrm{h} \\
(\AA)\end{array}$ & $\begin{array}{c}\mathrm{E}_{g} \\
(\mathrm{eV})\end{array}$ & $\begin{array}{c}\mathrm{Q} \\
(\mathrm{e})\end{array}$ \\
\hline $\mathrm{COCH} 2$ & -1.96 & 1.291 & 2.41 & 0.14 \\
$\mathrm{CO} 2 \mathrm{CH} 2$ & -1.31 & 1.487 & 2.21 & 0.47 \\
$\mathrm{NH} 3 \mathrm{CH} 2$ & -0.26 & 3.317 & 1.60 & 0.03 \\
\hline \hline
\end{tabular}

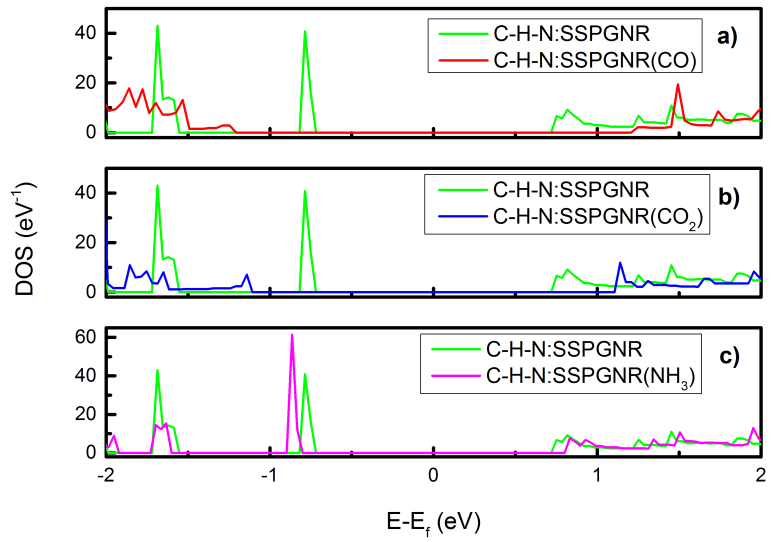

Figure 3: Density of states of systems: a) $\mathrm{C}-\mathrm{H}-\mathrm{N}: \operatorname{SSPGNR}(\mathrm{CO})$ and C-H-N:SSPGNR; b) C$\mathrm{H}-\mathrm{N}$ :SSPGNR $\left(\mathrm{CO}_{2}\right)$ and C-H-N:SSPGNR; c) C-H$\mathrm{N}: \mathrm{SSPGNR}\left(\mathrm{NH}_{3}\right)$ and C-H-N:SSPGNR.

the bond angles $\left(\beta_{1}, \beta_{2}, \beta_{3}\right)$ close to the edge of C-H-N:SSPGNR substrate were also changed after the gas molecule adsorption. The changes in the structural parameters due to this interaction is the basis of the change in electronic properties, which will be analyzed in the next section.

\section{iii Electronic Properties}

The electronic properties of C-H-N:SSPGNR were studied to understand its capacity to detect $\mathrm{CO}$, $\mathrm{CO}_{2}$ and $\mathrm{NH}_{3}$. We first investigated the density of states (DOS) of $\mathrm{CO}, \mathrm{CO}_{2}$ and $\mathrm{NH}_{3}$-adsorbed C-H$\mathrm{N}: S S P G N R$, as shown in Fig. 3. We also see that after the adsorption of gas molecules, the DOS of systems changed, and the band gaps of all three samples expanded. Specifically, the band gap of CO-adsorbed C-H-N:SSPGNR showed the largest increase; its band gap increased to $2.41 \mathrm{eV}$ while the band gap of C-H-N:SSPGNR was only $1.44 \mathrm{eV}$. On the other hand, the sample with the smallest band gap change was the $\mathrm{NH}_{3}$-adsorbed C-H-N:SSPGNR sample (Table 2). The trend seen in the calculated charge transfer $(\mathrm{Q})$ in Table 2 can be understood as capacity for relative electron donation or electron withdrawal of the adsorbed molecules. The positive $Q$ values mean that charge was transferred from the adsorbed molecules to the C-H-N:SSPGNR in all three cases, the charge transferred from the $\mathrm{CO}_{2}$ to the C-H-N:SSPGNR being the largest $(Q=0.47 e)$. 
Papers in Physics, vol. 13, ART. 130003 (2021) / N. T. Tien et al.

Table 3: Bond lengths of gas molecules before and after adsorption $\left(l_{1}\right.$ to $\left.l_{6}\right)$, bond angles of gas molecules before and after adsorption ( $\alpha_{1}$ to $\alpha_{4}$ ), bond lengths at edge of C-H-N:SSPGNR before and after gas molecule adsorption $\left(d_{1}\right.$ to $\left.d_{4}\right)$, and bond angles of C-H-N:SSPGNR before and after adsorption for gas molecules ( $\beta_{1}$ to $\left.\beta_{4}\right)$.

\begin{tabular}{lccccccc}
\hline \hline Samples & C-H-N:SSPGNR & $\mathrm{CO}$ & $\mathrm{CO}_{2}$ & $\mathrm{NH}_{3}$ & $\mathrm{COCH} 2$ & $\mathrm{CO} 2 \mathrm{CH} 2$ & NH3CH2 \\
\hline$l_{1}(\AA)$ & - & 1.128 & - & - & 1.184 & - & - \\
$l_{2}(\AA)$ & - & - & 1.128 & - & - & 1.213 & - \\
$l_{3}(\AA)$ & - & - & 1.128 & - & - & 1.213 & - \\
$l_{4}(\AA)$ & - & - & - & 1.163 & - & - & 1.022 \\
$l_{5}(\AA)$ & - & - & - & 1.163 & - & - & 1.026 \\
$l_{6}(\AA)$ & - & - & - & 1.163 & - & - & 1.029 \\
$\alpha_{1}\left({ }^{\circ}\right)$ & - & - & 180 & - & - & 122 & - \\
$\alpha_{2}\left(^{\circ}\right)$ & - & - & - & 107.8 & - & - & 106.0 \\
$\alpha_{3}\left(^{\circ}\right)$ & - & - & - & 107.8 & - & - & 106.1 \\
$\alpha_{4}\left({ }^{\circ}\right)$ & - & - & - & 107.8 & - & - & 106.5 \\
$d_{1}(\AA)$ & 1.338 & - & - & - & 1.339 & 1.366 & 1.338 \\
$d_{2}(\AA)$ & 1.540 & - & - & - & 1.397 & 1.425 & 1.536 \\
$d_{3}(\AA)$ & 1.508 & - & - & - & 1.414 & 1.409 & 1.507 \\
$\beta_{1}\left({ }^{\circ}\right)$ & 122.5 & - & - & - & 125.4 & 132.6 & 123.1 \\
$\beta_{2}\left({ }^{\circ}\right)$ & 108.5 & - & - & - & 102.9 & 108.6 & 108.8 \\
$\beta_{3}\left(^{\circ}\right)$ & 102.9 & - & - & - & 108.8 & 106.3 & 102.8 \\
$\beta_{4}\left({ }^{\circ}\right)$ & 120.4 & - & - & - & 131.2 & 111.4 & 120.2 \\
\hline \hline
\end{tabular}

To better understand the cause of the changes in band gaps after adsorption, we analyzed the contribution of the gas molecules by drawing the total density of states (TDOS) and the local density of states (LDOS), shown in Fig. 4. We also see that the main contribution to the changes in DOS is not due to the gas molecules but to changes in the substrate. In particular, the $\mathrm{CO}$ molecule has the most influence on DOS and the $\mathrm{NH}_{3}$ molecule has the least. In all cases, the major contribution to the changes in band gap width is the p orbitals of the atoms.

On the other hand, as can be see from Fig. 3 and Fig. 4, in all three cases there is an overlap between the DOS lines, which confirms the connection between the gas molecules and the substrate. However, in the case of $\mathrm{CO}$ and $\mathrm{CO}_{2}$ adsorption, there is an overlap and interlacing between TDOS and LDOS, so we can confirm that there is chemical bond formation. The adsorption in these two cases is chemical. In contrast, in the case of $\mathrm{NH}_{3}$ the adsorption is only the overlapping between TDOS and LDOS; $\mathrm{NH}_{3}$ can only physically adsorb on the edge of N:SSPGNR.

Figure 5 shows the electron density difference (EDD) for all three adsorbed samples. The EDD was calculated using the following formula:

$$
\Delta \rho=\rho_{(\text {Total })}-\rho_{(\text {ribbon })}-\rho_{(\text {gas })} .
$$

Here, $\rho_{(\text {Total })}$ and $\rho_{(\text {ribbon })}$ represent the total electron densities of the N:SSPGNR with and without adsorbed gas molecules, respectively, and $\rho_{\text {(gas) }}$ is the electron density of the isolated gas molecules.

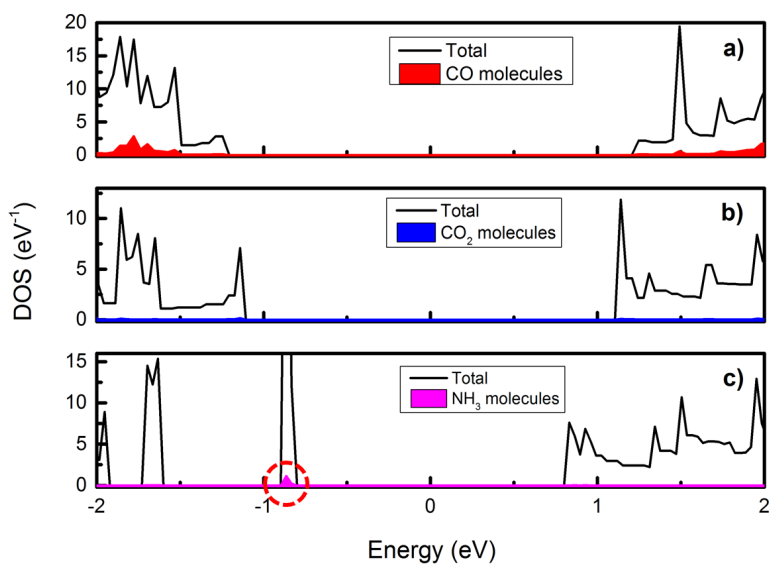

Figure 4: TDOS of systems: a) C-H-N:SSPGNR(CO); b) C-H-N:SSPGNR $\left(\mathrm{CO}_{2}\right)$; c) C-H-N:SSPGNR $\left(\mathrm{NH}_{3}\right)$ and LDOS of the gas molecules (the filled area under the DOS curve). 
a)
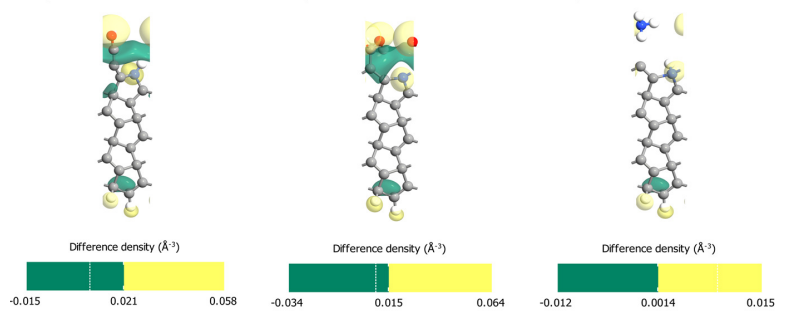

Figure 5: The electron density difference for $\mathrm{CO}, \mathrm{CO}_{2}$ and $\mathrm{NH}_{3}$-adsorbed N:SSPGNR. The isosurface value is taken as $0.009 \mathrm{eV} \AA^{-3}$. The electron density difference is defined as the valence electron density minus the neutral atom electron density.

It can be seen from the EDD plots that the charges were accumulated over the adsorbed gas molecules.

The formation of chemical bonds is evident in the case of $\mathrm{CO}$ and $\mathrm{CO}_{2}$ adsorption. The electron density at the interface region between $\mathrm{CO}$, $\mathrm{CO}_{2}$ and N:SSPGNR indicates that the adsorbed gas molecule does form covalent bonds with the $\mathrm{N}$ :SSPGNR after the adsorption process. In contrast, in the case of $\mathrm{NH}_{3}$ adsorption, no formation of covalent bonds was found, because there is little electron density difference at the interface between the $\mathrm{NH}_{3}$ gas molecule and N:SSPGNR.

\section{iv Transport Properties}

In the previous section, we showed that the adsorption of gas molecules causes changes in the electronic band gaps of N:SSPGNR. For further verification of $\mathrm{CO}, \mathrm{CO}_{2}$ and $\mathrm{NH}_{3}$ detection by $\mathrm{N}$ :SSPGNR, we studied the transport properties of C-H-N:SSPGNR before and after the adsorption of gas molecules. The current voltage (I-V) characteristics were obtained using a two-probe model.

Figure 6 shows the correlation between the current and the bias voltage of the adsorbed samples and the pure sample. We can see that all of these lines have the same shape. On the graph there is a conduction pause although the bias voltage increases. When the bias voltage reaches a certain limit value $(1.6 \mathrm{~V})$, the current begins to increase, then decreases until saturation at 2.0 V. Specifically, except for the $\mathrm{CO}_{2}$ adsorption sample, the current of the remaining samples starts to increase at the bias voltage of $1.6 \mathrm{~V}$ (threshold bias). For semiconductors, when the polarizing voltage is not

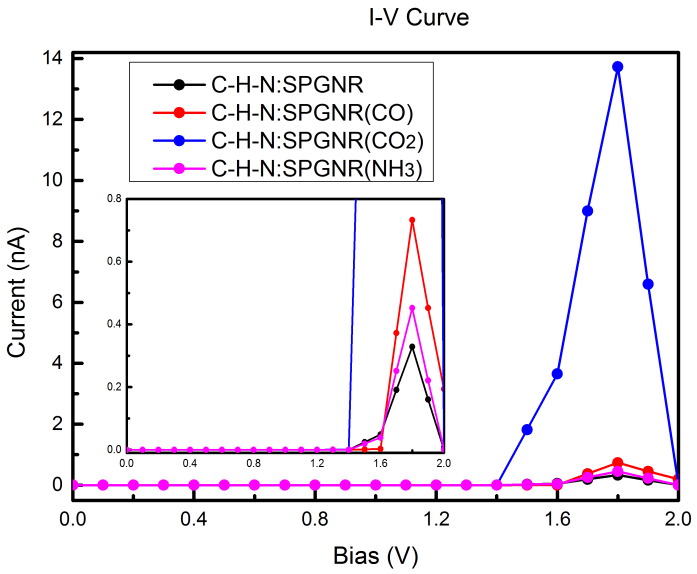

Figure 6: Calculated I-V curves for four structures: C-H-N:SSPGNR, C-H-N:SSPGNR(CO), C-H$\mathrm{N}: \operatorname{SSPGNR}\left(\mathrm{CO}_{2}\right)$ and C-H-N:SSPGNR$\left(\mathrm{NH}_{3}\right)$.

large enough the device stops conducting, but when it is large enough for an electron to cross the barrier, the device begins to conduct electricity. Thus, we can conclude that the structures under consideration are semiconductors. The maximum current obtained for pure C-H-N:SSPGNR, CO-adsorbed C-H-N:SSPGNR, $\mathrm{CO}_{2}$-adsorbed C-H-N:SSPGNR, and $\mathrm{NH}_{3}$-adsorbed C-H-N:SSPGNR is $0.3 \mathrm{nA}, 0.7$ $\mathrm{nA}, 13.7 \mathrm{nA}$, and $0.4 \mathrm{nA}$, respectively. In all four cases, the maximum currents occur at the bias voltage of $1.8 \mathrm{~V}$. The maximum current of the $\mathrm{CO}_{2}$ adsorption sample is the highest, 45 times greater than that of the pure sample. The maximum current of the $\mathrm{NH}_{3}$ adsorption sample is the lowest, only 1.3 times greater than that of the pure sample. All these findings suggest that the N:SSPGNR current can be distinguished before and after molecule adsorption [42].

To explain the change in the trend of the I-V curves in Fig. 6, we considered the bias dependent transmission spectra, $\mathrm{T}(\mathrm{E}, \mathrm{Vb})$, of the four studied structures via Fig. 7 (left-hand panels). We see that the $\mathrm{T}(\mathrm{E}, \mathrm{Vb})$ of the pure C-H-N:SSPGNR, the CO-adsorbed C-H-N:SSPGNR, and the $\mathrm{NH}_{3}-$ adsorbed C-H-N:SSPGNR differ very little. However, the $\mathrm{T}(\mathrm{E}, \mathrm{Vb})$ spectrum of the $\mathrm{CO}_{2}$-adsorbed $\mathrm{C}-\mathrm{H}-\mathrm{N}$ :SSPGNR is very different from the three other samples. This is also related to the charge transfer phenomenon mentioned in Table 2. Furthermore, the various maximum currents occur at 
a)

c)
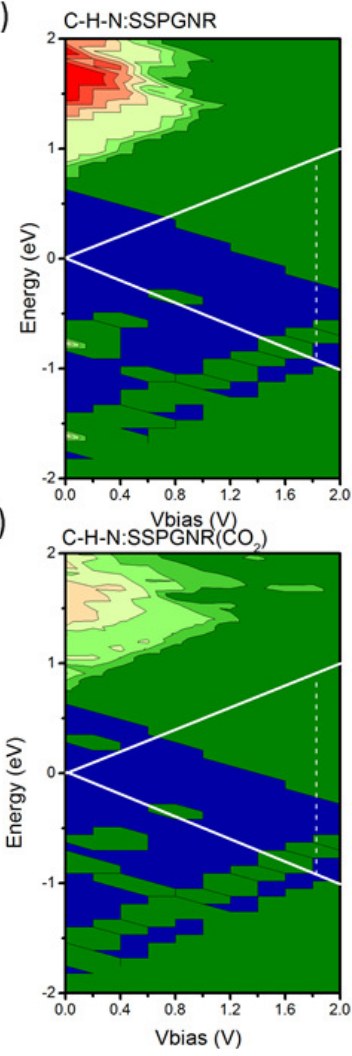

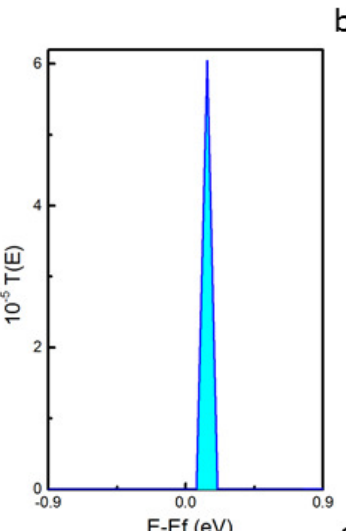

b) C-H-N:SSPGNR(CO)

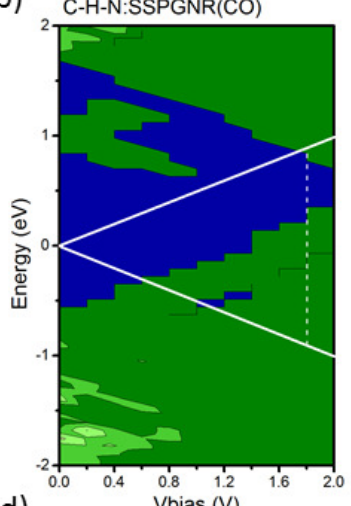

d) $\mathrm{C}$-H-N:SSPGNR(N)

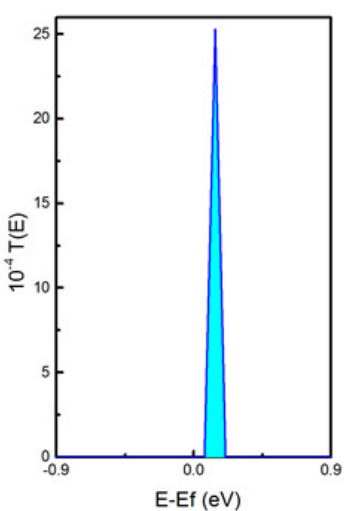

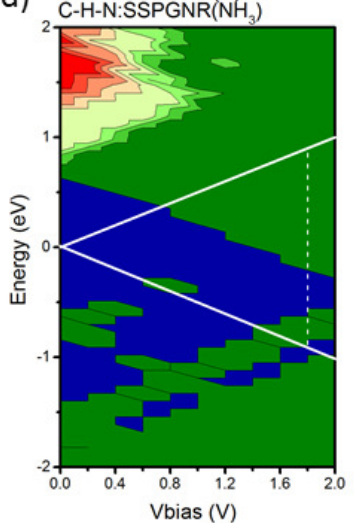
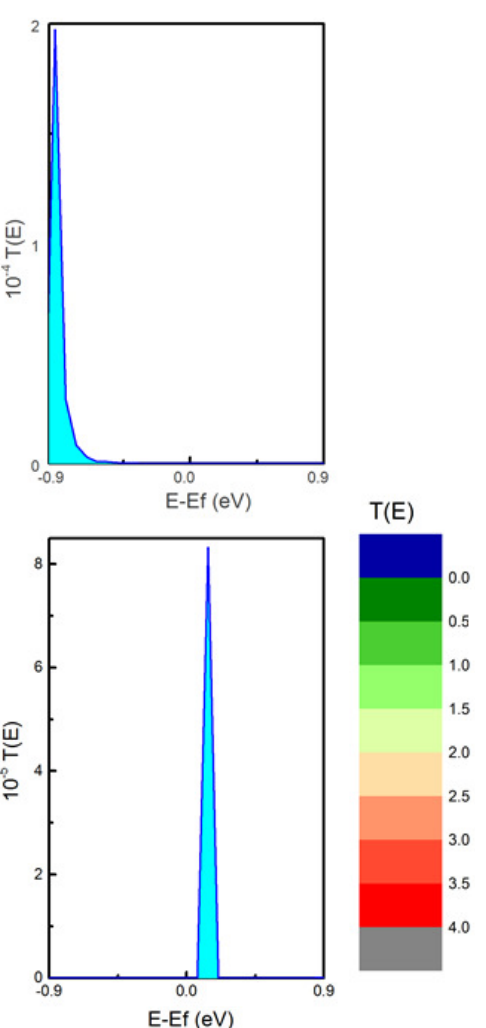

Figure 7: Left-hand panels: The contour of the bias-dependent transmission $\mathrm{T}(\mathrm{E}, \mathrm{Vb})$; Right-hand panels: Transmission coefficient at $1.8 \mathrm{~V}$ bias of four structures: a) C-H-N:SSPGNR, b) C-H-N:SSPGNR(CO), c) C-H$\mathrm{N}: \operatorname{SSPGNR}\left(\mathrm{CO}_{2}\right)$ and d) C-H-N:SSPGNR$\left(\mathrm{NH}_{3}\right)$.

the bias voltage of $1.8 \mathrm{~V}$; the transmission coefficients at $1.8 \mathrm{~V}$ bias of the four structures were calculated and shown in the right-hand panels of Fig. 7. The values and filled zones bounded by the horizontal axis and $\mathrm{T}(\mathrm{E}, \mathrm{Vb}=1.8 \mathrm{~V})$ curves help us to explain the changing tendency of the maximum currents of the four structures.

\section{Conclusions}

In summary, using first-principle calculations we studied the adsorption geometry, adsorption energy, charge transfer, density of states, partial density of states and I-V curves of N:SSPGNR with gas molecule $\left(\mathrm{CO}, \mathrm{CO}_{2}\right.$ and $\left.\mathrm{NH}_{3}\right)$ adsorption. Our calculated results show that edge adsorption of $\mathrm{CO}$ and $\mathrm{CO}_{2}$ molecules is more energetically favorable than edge adsorption of $\mathrm{NH}_{3}$. Moreover, the adsorption of $\mathrm{CO}$ and $\mathrm{CO}_{2}$ on the edge of N:SSPGNR is chemical, while the adsorption of $\mathrm{NH}_{3}$ is physical. The current voltage $(\mathrm{I}-\mathrm{V})$ characteristics were also investigated using the non-equilibrium Green's function (NEGF) approach. The results indicate that conductance of the $\mathrm{CO}, \mathrm{CO}_{2}$, and $\mathrm{NH}_{3}$ adsorption N:SSPGNR can be distinguished at the bias voltage of $1.8 \mathrm{~V}$. These changes in electronic and transport properties make N:SSPGNR a promising candidate for gas detector development.

Acknowledgements - This research was funded in part by the Can Tho University Improvement Project VN14-P6, supported by a Japanese ODA loan. We are grateful to the information center and network administrator of Can Tho University (CTU) for computational support. We also thank Prof. Yoshitada Morikawa (Osaka University) for discussing research ideas. 
Papers in Physics, vol. 13, ART. 130003 (2021) / N. T. Tien et al.

[1] N Haleh, J Aashish, P Jaewoo, E Arezoo, Advanced micro-and nano-gas sensor technology: A review, Sensors 19, 1285 (2019).

[2] S Yang, C Jiang, S H Wei, Gas sensing in 2D materials, Appl. Phys. Rev. 4, 021304 (2017).

[3] C Tan, X Cao, X J Wu, Q He, J Yang, H Zhang, et. al, Recent advances in ultrathin two-dimensional nanomaterials, Chem. Rev. 117, 6225 (2017).

[4] J Chen, L Xu, W Li, X Gou, $\alpha$-Fe2O3 nanotubes in gas sensor and lithium-ion battery applications, Adv. Mater. 17, 582 (2005).

[5] V Singh, D Joung, L Zhai, S Das, S I Khondaker, S Seal, Graphene based materials: Past, present and future, Prog. Mater. Sci. 56, 1178 (2011).

[6] P Sun, Z Kunlin, H Wang, Recent developments in graphene-based membranes: Structure, mass-transport mechanism and potential applications, Adv. Mater. 28, 2287 (2016).

[7] D N Quang, L Tuan, N T Tien, Electron mobility in Gaussian heavily doped ZnO surface quantum wells, Phys. Rev. B 77, 125326 (2008).

[8] B Huang, Z Li, Z Liu, G Zhou, S Hao, J Wu, B L Gu, W Duan, Adsorption of gas molecules on graphene nanoribbons and its implication for nanoscale molecule sensor, J. Phys. Chem. C 112, 13442 (2008).

[9] F Schwierz, Graphene transistors, Nature Nanotech. 5, 487 (2010).

[10] A H Castro Neto, F Guinea, N M R Peres, K $\mathrm{S}$ Novoselov, A K Geim, The electronic properties of graphene, Rev. Mod. Phys. 81, 109 (2009).

[11] F Bonaccorso, Z Sun, T Hasan, A Ferrari, Graphene photonics and optoelectronics, Nature photon. 4, 611 (2010).

[12] P Avouris, Graphene: Electronic and photonic properties and devices, Nano Lett. 10, 4285 (2010).
[13] P Vogt, P D Padova, C Quaresima, J Avila, E Frantzeskakis, M C Asensio, A Resta, B Ealet, G L Lay, Silicene: Compelling experimental evidence for graphenelike two-dimensional silicon, Phys. Rev. Lett. 108, 155501 (2012).

[14] A Ziletti, A Carvalho, D K Campbell, D F Coker, A H Castro Neto, Oxygen defects in phosphorene, Phys. Rev. Lett. 114, 046801 (2015).

[15] X Deng, K Yao, K Sun, W X Li, J Lee, C Matranga, Growth of single-and bilayer $\mathrm{ZnO}$ on $A u(111)$ and interaction with copper, J. Phys. Chem. C 117, 11211 (2013).

[16] X Blase, A Rubio, S G Louie, M L Cohen, Quasiparticle band structure of bulk hexagonal boron nitride and related systems, Phys. Rev. B 51, 6868 (1995).

[17] D W Latzke, W Zhang, A Suslu, T R Chang, H Lin, H T Jeng, S Tongay, J Wu, A Bansil, A Lanzara, Electronic structure, spin-orbit coupling, and interlayer interaction in bulk $\mathrm{MoS}_{2}$ and $W S_{2}$, Phys. Rev. B 91, 235202 (2015).

[18] S Zhang, J Zhou, Q Wang, X Chen, Y Kawazoe, P Jena, Penta-graphene: A new carbon allotrope, P. Natl. Acad. Sci. U.S.A. 112, 2372 (2015).

[19] T Stauber, J I Beltran, J Schliemann, Tightbinding approach to penta-graphene, Sci. Rep. 6, 1 (2016).

[20] T Y Mi, N D Khanh, R Ahuja, N T Tien, Diverse structural and electronic properties of pentagonal $\mathrm{SiC}_{2}$ nanoribbons: $A$ firstprinciples study, Mater. Today Comm. 26, 102047 (2021).

[21] J Sun, Y Guo, Q Wang, Y Kawazoe, Thermal transport properties of penta-graphene with grain boundaries, Carbon 145, 445 (2019).

[22] H Einollahzadeh, R Dariani, S Fazeli, Computing the band structure and energy gap of pentagraphene by using DFT and $G_{0} W_{0}$ approximations, Solid State Comm. 229, 1 (2016). 
Papers in Physics, vol. 13, ART. 130003 (2021) / N. T. Tien et al.

[23] X Wu, V Varshney, J Lee, T Zhang, J L Wohlwend, A K Roy, T Luo, Hydrogenation of penta-graphene leads to unexpected large improvement in thermal conductivity, Nano Lett. 16, 3925 (2016).

[24] S Winczewski, J Rybicki, Anisotropic mechanical behavior and auxeticity of penta-graphene: Molecular statics/molecular dynamics studies, Carbon 146, 572 (2019).

[25] R Krishnan, W S Su, H T Chen, A new carbon allotrope: Penta-graphene as a metal-free catalyst for CO oxidation, Carbon 114, 465 (2017).

[26] H Qin, C Feng, X Luan, D Yang, Firstprinciples investigation of adsorption behaviors of small molecules on penta-graphene, Nanoscale Res. Lett. 13, 1 (2018).

[27] C P Zhang, B Li, Z G Shao, First-principle investigation of $\mathrm{CO}$ and $\mathrm{CO}_{2}$ adsorption on Fe-doped penta-graphene, Appl. Surf. Sci. 469, 641 (2019).

[28] P F Yuan, Z H Zhang, Z Q Fan, M Qiu, Electronic structure and magnetic properties of penta-graphene nanoribbons, Phys. Chem. Chem. Phys. 19, 9528 (2017).

[29] C He, X F Wang, W X Zhang, Coupling effects of the electric field and bending on the electronic and magnetic properties of pentagraphene nanoribbons, Phys. Chem. Chem. Phys. 19, 18426 (2017).

[30] N T Tien, V T Phuc, R Ahuja, Tuning electronic transport properties of zigzag graphene nanoribbons with silicon doping and phosphorus passivation, AIP Adv. 8, 085123 (2018).

[31] N T Tien, P T B Thao, V T Phuc, R Ahuja, Electronic and transport features of sawtooth penta-graphene nanoribbons via substitutional doping, Physica E: Low Dimens. Syst. Nanostruct. 114, 113572 (2019).

[32] N T Tien, P T B Thao, V T Phuc, R Ahuja, Influence of edge termination on the electronic and transport properties of sawtooth penta- graphene nanoribbons, J. Phys. Chem. Solids 146, 109528 (2020).

[33] Y H Li, P F Yuan, Z Q Fan, Z H Zhang, Electronic properties and carrier mobility for penta-graphene nanoribbons with nonmetallicatom-terminations, Org. Electron. 59, 306 (2018).

[34] T Y Mi, D M Triet, N T Tien, Adsorption of gas molecules on penta-graphene nanoribbon and its implication for nanoscale gas sensor, Physics Open 2, 100014 (2020).

[35] A Saffarzadeh, Modeling of gas adsorption on graphene nanoribbons, J. Appl. Phys. 107, 114309 (2010).

[36] J Taylor, H Guo, J Wang, Ab initio modeling of quantum transport properties of molecular electronic devices, Phys. Rev. B 63, 245407 (2001).

[37] M Brandbyge, J L Mozos, P Ordejón, J Taylor, K Stokbro, Density-functional method for nonequilibrium electron transport, Phys. Rev. B 65, 165401 (2002).

[38] J Zhao, A Buldum, J Han, J P Lu, Gas molecule adsorption in carbon nanotubes and nanotube bundles, Nanotechnology 13, 195 (2002).

[39] J W Feng, Y J Liu, H X Wang, J X Zhao, Q H Cai, X Z Wang, Gas adsorption on silicene: A theoretical study, Comp. Mater. Sci. 87, 218 (2014).

[40] P J Perdew, K Burke, M Ernzerhof, Generalized gradient approximation made simple, Physical Rev. Lett. 77, 3865 (1996).

[41] P Pyykkö, M Atsumi, Molecular single-bond covalent radii for elements 1-118, Chem. Eur. J. 15, 186 (2009).

[42] L Tang, M Q Cheng, Q Chen, T Huang, K Yang, W Q Huang, W Hu, G F Huang, Ultrahigh sensitivity and selectivity of pentagonal $\mathrm{SiC}_{2}$ monolayer gas sensors: The synergistic effect of composition and structural topology, Phys. Status Solidi B 257, 1900445 (2020). 